\title{
Cauchy Problem for a Darboux Integrable Wave Map System and Equations of Lie Type
}

\author{
Peter J. VASSILIOU
}

Program in Mathematics and Statistics, University of Canberra, 2601 Australia

E-mail: peter.vassiliou@canberra.edu.au

Received September 27, 2012, in final form March 12, 2013; Published online March 18, 2013

http://dx.doi.org/10.3842/SIGMA.2013.024

\begin{abstract}
The Cauchy problem for harmonic maps from Minkowski space with its standard flat metric to a certain non-constant curvature Lorentzian 2-metric is studied. The target manifold is distinguished by the fact that the Euler-Lagrange equation for the energy functional is Darboux integrable. The time evolution of the Cauchy data is reduced to an ordinary differential equation of Lie type associated to SL(2) acting on a manifold of dimension 4. This is further reduced to the simplest Lie system: the Riccati equation. Lie reduction permits explicit representation formulas for various initial value problems. Additionally, a concise (hyperbolic) Weierstrass-type representation formula is derived. Finally, a number of open problems are framed.
\end{abstract}

Key words: wave map; Cauchy problem; Darboux integrable; Lie system; Lie reduction; explicit representation

2010 Mathematics Subject Classification: 53A35; 53A55; 58A15; 58A20; 58A30

This paper is dedicated to Peter Olver on the occasion of his 60th birthday in celebration of his contributions to mathematics; especially his influential, diverse applications of Lie theory.

\section{Introduction}

Let $(M, g)$ and $(N, h)$ be Riemannian or pseudo-Riemannian manifolds and $\varphi: M \rightarrow N$ a smooth map. The energy of $\varphi$ over a compact domain $\mathcal{D} \subseteq M$ is

$$
e(\varphi)=\frac{1}{2} \int_{\mathcal{D}} g^{i j}(x) h_{\alpha \beta}(\varphi) \frac{\partial \varphi^{\alpha}}{\partial x_{i}} \frac{\partial \varphi^{\beta}}{\partial x_{j}} d \operatorname{vol}_{M}
$$

The critical points of $e(\varphi)$ satisfy the partial differential equation (PDE)

$$
\triangle \varphi^{\gamma}+g^{i j} \Lambda_{\alpha \beta}^{\gamma} \frac{\partial \varphi^{\alpha}}{\partial x_{i}} \frac{\partial \varphi^{\beta}}{\partial x_{j}}=0
$$

where $\triangle$ is the Laplacian on $M$ and $\Lambda_{\alpha \beta}^{\gamma}$ the Christoffel symbols on $N$. A map $\varphi$ is said to be harmonic if it is critical for $e(\varphi)$. Harmonic maps generalise harmonic functions and geodesics and have been under intense study since the pioneering work of Eells and Sampson [8]; see [3] and [12] and references therein for comprehensive introductions to the field. If domain $(\varphi)=$ $M=\mathbb{R}$ then harmonic maps are geodesic flows. If $\operatorname{codomain}(\varphi)=N=\mathbb{R}$ then harmonic maps are harmonic functions. If $(M, g)$ is pseudo-Riemannian then harmonic maps are called wave maps. In this paper our focus is on wave maps, specifically, the case $(M, g)=\left(\mathbb{R}^{1,1}, d x d y\right)$. Any further reference to wave maps in this paper means the domain space $M$ is Minkowski space $\mathbb{R}^{1,1}$

\footnotetext{
${ }^{\star}$ This paper is a contribution to the Special Issue "Symmetries of Differential Equations: Frames, Invariants and Applications". The full collection is available at http://www.emis.de/journals/SIGMA/SDE2012.html
} 
with its standard flat metric. To provide slightly more insight, a "physical" illustration of wave maps in this class can be given: the motion of a frictionless elastic string constrained to vibrate on Riemannian surface $(N, h)$, such as a sphere, is exactly described by a wave map into $N$; see [17].

There is a well-known geometric literature on wave maps that has developed over the last two decades, especially their existence as solutions of completely integrable systems; see [11] for a textbook account with many references. There is a closely related physics literature where the relevant systems are known as nonlinear sigma models; see [22]. The first person to treat the Cauchy problem for wave maps into Riemannian targets was Chao-Hao Gu [10]. He established the fundamental result that for smooth initial data, wave maps into complete Riemannian metrics have long-time existence. Gu's work initiated many further investigations where regularity constraints on the initial data have been significantly relaxed. Furthermore some higher dimensional problems have been treated; see [16].

In this paper we initiate the study of the Cauchy problem for wave maps in the special case where the systems they satisfy are Darboux integrable. Our first main result proves that the solution of the Cauchy problem for such a Darboux integrable nonlinear sigma model can be quite explicitly expressed as the flow of a special vector field $\xi_{k_{1}, k_{2}}$ (see Theorem 1 ) which itself is a curve in a certain Lie algebra of vector fields canonically and intrinsically associated to the Darboux integrable nonlinear sigma model, namely, its Vessiot algebra. In consequence of this, standard constructions which facilitate the resolution of systems of Lie type such as Lie reduction become available to the solution of the Cauchy problem for such wave map systems. For this reason we have included an appendix to this paper which gives a brief summary of the main results on systems of Lie type adapted to the applications we envisage. In this paper we have decided to focus on just one interesting nonlinear sigma model in order to discuss the relationship between Darboux integrable hyperbolic systems on the one hand and the resolution of the corresponding Cauchy problem via differential systems of Lie type and to do so as explicitly as possible. However, it will be seen that the proof of Theorem 1 is easy to generalise to other Darboux integrable systems. Indeed the very recent work [1] outlines a general, intrinsic proof of the close relationship between systems of Lie type and the Cauchy problem for a wide class of Darboux integrable exterior differential systems. The second main result of the paper marshalls the general theory of Darboux integrable exterior differential systems [2], and generalised Goursat normal form $[18,19]$ to derive a hyperbolic Weierstrass-type representation (Theorem 3) for wave maps into the non-constant curvature metric (1).

As the name implies the notion of Darboux integrability originated in the 19th century and was most significantly developed by Goursat [9]. Classically, it was a method for constructing the "general solution" of second order PDE in one dependent and two independent variables

$$
F\left(x, y, u, u_{x}, u_{y}, u_{x x}, u_{x y}, u_{y y}\right)=0
$$

that generalised the so called "method of Monge". It relies on the notion of characteristics and their first integrals. We refer the reader to [9, 13, 20, 21] for further information on classical Darboux integrability. There are also extensive studies of Darboux integrable systems relevant to the equation class under study in the works [14] and [23].

In this paper we use a new geometric formulation of Darboux integrable exterior differential systems [2]. At the heart of this theory is the fundamental notion of a Vessiot group which, together with systems of Lie type are our main tools for the study of the Cauchy problem for wave maps.

The PDE that govern wave maps have the semilinear form

$$
\boldsymbol{u}_{x y}=\boldsymbol{f}\left(x, y, \boldsymbol{u}, \boldsymbol{u}_{x}, \boldsymbol{u}_{y}\right), \quad \boldsymbol{u}, \boldsymbol{f} \in \mathbb{R}^{n} .
$$

Each solution possesses a double foliation of curves called characteristics. Such PDE often model wave-like phenomena and projection of these curves into the independent variable space 
describes the space-time history of the wave propagation. The characteristics of $\boldsymbol{u}_{x y}=\boldsymbol{f}$ are the integral curves of a pair of rank $n+1$ distributions

$$
H_{1}=\left\{D_{x}+D_{y} \boldsymbol{f} \cdot \partial_{\boldsymbol{u}_{y y}}, \partial_{\boldsymbol{u}_{x x}}\right\}, \quad H_{2}=\left\{D_{y}+D_{x} \boldsymbol{f} \cdot \partial_{\boldsymbol{u}_{x x}}, \partial_{\boldsymbol{u}_{y y}}\right\}
$$

where

$$
D_{x}=\partial_{x}+\boldsymbol{u}_{x} \partial_{\boldsymbol{u}}+\boldsymbol{u}_{x x} \partial_{\boldsymbol{u}_{x}}+\boldsymbol{f} \partial_{\boldsymbol{u}_{y}}, \quad D_{y}=\partial_{y}+\boldsymbol{u}_{y} \partial_{\boldsymbol{u}}+\boldsymbol{f} \partial_{\boldsymbol{u}_{x}}+\boldsymbol{u}_{y y} \partial_{\boldsymbol{u}_{y}},
$$

are the total differential operators along solutions of the PDE. Note that if $\boldsymbol{\theta}$ is the standard Cartan codistribution for $\boldsymbol{u}_{x y}=\boldsymbol{f}$ then $H_{1} \oplus H_{2}=\operatorname{ann} \boldsymbol{\theta}$. Distributions $H_{i}$ are well-defined with canonical structure.

Definition 1. If $\Delta$ is a distribution on manifold $M$ then a function $f: M \rightarrow \mathbb{R}$ is said to be a first integral of $\Delta$ if $X f=0$ for all $X \in \Delta$.

There is a geometric definition of Darboux integrable exterior differential system [2]. For wave map equations it reduces to

Definition 2. A semilinear system $\boldsymbol{u}_{x y}=\boldsymbol{f}$ with $\boldsymbol{u}, \boldsymbol{f} \in \mathbb{R}^{n}$ is Darboux integrable at a given order if each of its characteristic systems $H_{i}$ has at least $n+1$ independent first integrals at that order.

\section{The Cauchy problem}

We consider wave maps

$$
\boldsymbol{u}:\left(\mathbb{R}^{1,1}, d x d y\right) \rightarrow\left(\mathbb{R}^{2}, \frac{d u_{1}^{2}-d u_{2}^{2}}{1+e^{-u_{1}}}\right) .
$$

R. Ream [15] studied the PDE for wave maps into nonzero curvature surface metrics that are Darboux integrable on the 2- and 3-jets and proved a theorem that any such metric is (real) equivalent to one or other of the metrics

$$
\rho_{ \pm}:=\frac{d u_{1}^{2}+d u_{2}^{2}}{1 \pm e^{u_{1}}}
$$

Here we consider a semi-Riemannian version of a Ream metric and study the corresponding Cauchy problem. We show how the solution of the Cauchy problem for wave maps (1) can be expressed as an ordinary differential equation of Lie type. Indeed we prove that the solution of the Cauchy problem for wave maps is naturally equivalent to an initial value problem of a Lie system for a local action of SL(2) on a manifold that is locally diffeomorphic to $\mathbb{R}^{4}$. This is further reduced to an initial value problem for a single Riccati equation together with a quadrature.

The target metric in (1) does not have constant curvature nevertheless is globally defined and positively curved everywhere; in fact $K=2^{-1}\left(1+e^{u_{1}}\right)^{-1}$. However, the metric is nonetheless very special because the wave map system turns out to be Darboux integrable, as demonstrated in $[15]$.

The Lagrangian density for this metric is

$$
\mathcal{L}=\frac{u_{1 x} u_{1 y}-u_{2 x} u_{2 y}}{1+e^{-u_{1}}}
$$

whose Euler-Lagrange equation is

$$
u_{1 x y}+\frac{u_{1 x} u_{1 y}+u_{2 x} u_{2 y}}{2\left(1+e^{u_{1}}\right)}=0, \quad u_{2 x y}+\frac{u_{1 x} u_{2 y}+u_{2 x} u_{1 y}}{2\left(1+e^{u_{1}}\right)}=0 .
$$


The change of variables $\left(u_{1}, u_{2}\right) \mapsto\left(\left(u_{1}+u_{2}\right) / 2,\left(u_{1}-u_{2}\right) / 2\right)=(u, v)$ transforms this to

$$
u_{x y}+\frac{u_{x} u_{y}}{2\left(1+e^{u / 2+v / 2}\right)}=0, \quad v_{x y}+\frac{v_{x} v_{y}}{2\left(1+e^{u / 2+v / 2}\right)}=0 .
$$

We now prove

Theorem 1. Consider the initial value problem

$$
\begin{aligned}
& u_{x y}+\frac{u_{x} u_{y}}{2\left(1+e^{u / 2+v / 2}\right)}=0, \quad v_{x y}+\frac{v_{x} v_{y}}{2\left(1+e^{u / 2+v / 2}\right)}=0, \\
& u_{\left.\right|_{\gamma}}=\phi_{1}, \quad v_{\left.\right|_{\gamma}}=\phi_{2}, \quad \frac{\partial u}{\partial \boldsymbol{n}_{\left.\right|_{\gamma}}}=\psi_{1}, \quad \frac{\partial v}{\partial \boldsymbol{n}_{\left.\right|_{\gamma}}}=\psi_{2},
\end{aligned}
$$

where $\gamma$ is a curve with tangents nowhere parallel to the $x$-or $y$-axes, $\boldsymbol{n}$ is a unit normal vector field along $\gamma$ and $\phi_{i}, \psi_{i}$ are smooth functions along $\gamma$.

1. Problem (3) has a unique smooth local solution. Moreover, the unique local solution is expressible as the solution of an ordinary differential equation of Lie type associated an action of $\mathrm{SL}(2)$ on $\mathbb{R}^{4}$.

2. Given the unique local solution $(u, v)$ from part 1 , the Cauchy problem for harmonic maps

$$
\left(\mathbb{R}^{1,1}, d x d y\right) \rightarrow\left(M, \frac{d u_{1}^{2}-d u_{2}^{2}}{1+e^{-u_{1}}}\right)
$$

is given by

$$
u_{1}=u+v, \quad u_{2}=u-v .
$$

where $u_{1}, u_{2}$ satisfy initial conditions

$$
u_{\left.1\right|_{\gamma}}=\phi_{1}+\phi_{2}, \quad u_{\left.2\right|_{\gamma}}=\phi_{1}-\phi_{2},\left.\quad \frac{\partial u_{1}}{\partial \boldsymbol{n}}\right|_{\left.\right|_{\gamma}}=\psi_{1}+\psi_{2},\left.\quad \frac{\partial u_{2}}{\partial \boldsymbol{n}}\right|_{\left.\right|_{\gamma}}=\psi_{1}-\psi_{2} .
$$

Proof. By hyperbolicity, the problem is locally well-posed. System (2) has four first integrals on each characteristic system, $H_{1}, H_{2}$. Let us label the first integrals

$$
y, \beta_{1}, \beta_{2}, \beta_{3} \text { for } H_{1}, \quad \text { and } \quad x, \alpha_{1}, \alpha_{2}, \alpha_{3} \text { for } H_{2} \text {. }
$$

For this system $\alpha_{1}, \beta_{1}$ are first order differential functions while $\alpha_{2}, \alpha_{3}, \beta_{2}, \beta_{3}$ are of second order. Finally, while the 8 first integrals are functionally independent, we have

$$
\frac{d \alpha_{1}}{d x}=\alpha_{2} \quad \text { and } \quad \frac{d \beta_{1}}{d y}=\beta_{2} .
$$

Let $k_{1}(y), k_{2}(y)$ be arbitrary functions and consider the overdetermined PDE system defined by (2) together with the additional PDE

$$
\beta_{1}=k_{1}(y), \quad \beta_{2}=\dot{k}_{1}(y), \quad \beta_{3}=k_{3}(y),
$$

where the dot denotes $y$-differentiation. It can be shown that this overdetermined system $\mathcal{E}^{\prime}$ is involutive and moreover admits a 1-dimensional Cauchy distribution, as we will see. Now suppose we fix a smooth curve $\gamma$ embedded in a portion of the $x y$-plane, $\mathcal{N}$ and suppose Cauchy data is prescribed along $\gamma$ as in the Theorem statement. Then by an argument similar to [21, Proposition 3.3], $\gamma$ can be lifted to a unique curve $\widehat{\gamma}: I \subseteq \mathbb{R} \rightarrow J^{1}\left(\mathcal{N}, \mathbb{R}^{2}\right)$ which agrees with all the Cauchy data. Let $\iota: \mathcal{H}_{k_{1}, k_{2}} \rightarrow J^{2}\left(\mathcal{N}, \mathbb{R}^{2}\right)$ denote the submanifold in $J^{2}\left(\mathcal{N}, \mathbb{R}^{2}\right)$ defined 
by PDE system $\mathcal{E}^{\prime}$ and $\Theta$ the contact system on $J^{2}$. Let $\theta_{k_{1}, k_{2}}=\iota^{*} \Theta$ be the Pfaffian system whose integral submanifolds are the solutions of $\mathcal{E}^{\prime}$. Our aim is to choose the functions $k_{1}, k_{2}$, if possible, in order that we can extend $\widehat{\gamma}$ to a 1-dimensional integral submanifold $\widetilde{\gamma}$ of $\theta_{k_{1}, k_{2}}$.

Now

$$
\begin{aligned}
\theta_{k_{1}, k_{2}}=\left\{\omega^{1}=d u-u_{x} d x-u_{y} d y, \omega^{2}=d v-v_{x} d x-v_{y} d y, \omega^{3}=d u_{x}-u_{x x} d x-f^{1} d y,\right. \\
\left.\omega^{4}=d u_{y}-f^{1} d x-u_{y y} d y, \omega^{5}=d v_{x}-v_{x x} d x-f^{2} d y, \omega^{6}=d v_{y}-f^{2} d x-v_{y y} d y\right\} .
\end{aligned}
$$

Pulling back by $\widehat{\gamma}$ we observe that $\omega^{1}, \omega^{2}$ pullback to zero by construction. Forms $\omega^{4}$ and $\omega^{6}$ define the functions $u_{y y}$ and $v_{y y}$ along $\gamma$ while $\omega^{3}, \omega^{5}$ define functions $u_{x x}$ and $v_{x x}$ along $\gamma$. All these functions are expressed in terms of the Cauchy data, $\phi_{i}, \psi_{i}$. Substituting these back into (5) determines the functions $k_{1}, k_{2}$ in terms of $\phi_{i}, \psi_{i}$. For completeness we give the first integrals of $\mathrm{H}_{2}$

$$
\begin{aligned}
& x, \quad \alpha_{1}=\frac{u_{x} v_{x}}{1+\exp \left(-\frac{u+v}{2}\right)}, \quad \alpha_{2}=\frac{d \alpha_{1}}{d x}, \\
& \alpha_{3}=\frac{\left(2 v_{x} u_{x x}-2 u_{x} v_{x x}-u_{x} v_{x}^{2}+v_{x} u_{x}^{2}+2\left(v_{x} u_{x x}-u_{x} v_{x x}-u_{x} v_{x}^{2}+u_{x}^{2} v_{x}\right) \exp \left(\frac{u+v}{2}\right)\right)}{u_{x} v_{x}\left(1+\exp \left(\frac{u+v}{2}\right)\right)} .
\end{aligned}
$$

Those of $H_{1}$ are similar but with $y$ replacing $x$.

We will now use these first integrals to demonstrate that $\theta_{k_{1}, k_{2}}$ has a one-dimensional Cauchy distribution and that in particular the Cauchy vector can be chosen to be a curve in a certain Lie algebra - the Vessiot algebra [2] of system (2). It will be seen that the Cauchy vector is generically transverse to the Cauchy data and extends the one-dimensional integral submanifold of $\theta_{k_{1}, k_{2}}$ to the solution of the Cauchy problem. Because the Cauchy vector is a curve in a Lie algebra, this extension from a one-dimensional to a two-dimensional integral of $\theta_{k_{1}, k_{2}}$ is an ordinary differential equation $\mathfrak{L}$ of Lie type. Its coefficients and initial conditions are fixed by all the data present in the problem, including the Cauchy data. Any solution of $\mathfrak{L}$ (independently of its initial conditions) permits a Lie reduction of $\mathfrak{L}$ and will permit us to solve the IVP for $\mathfrak{L}$.

Indeed, setting $z_{1}=e^{u / 2}, z_{2}=e^{v / 2}, z_{3}=u_{y}, z_{4}=v_{x}, a_{i}=\alpha_{i}, b_{i}=\beta_{i}, i=1,2,3$ we calculate that the contact system on $J^{2}\left(\mathbb{R}^{2}, \mathbb{R}^{2}\right)$ pulled back to $\operatorname{PDE}(2)$ is $\Psi=\left\{\kappa^{1}, \ldots, \kappa^{6}\right\}$, where

$$
\begin{aligned}
& \kappa^{1}=d a_{1}-a_{2} d x, \quad \kappa^{2}=d b_{1}-b_{2} d y, \quad \kappa^{3}=d z_{1}-\frac{a_{1}\left(1+z_{1} z_{2}\right)}{2 z_{2} z_{4}} d x-\frac{z_{1} z_{3}}{2} d y, \\
& \kappa^{4}=d z_{2}-\frac{z_{2} z_{4}}{2} d x-\frac{b_{1}\left(1+z_{1} z_{2}\right)}{2 z_{1} z_{3}} d y, \quad \kappa^{5}=d z_{3}+\frac{a_{1} z_{3}}{2 z_{1} z_{2} z_{4}} d x+\frac{1}{2}\left(z_{3}^{2}-b_{3} z_{3}-b_{1}\right) d y, \\
& \kappa^{6}=d z_{4}+\frac{1}{2}\left(z_{4}^{2}-a_{3} z_{4}-a_{1}\right) d x+\frac{b_{1} z_{4}}{2 z_{1} z_{2} z_{3}} d y .
\end{aligned}
$$

Pulling $\Psi$ back to submanifold (5) yields a Pfaffian system with 1-dimensional Cauchy distribution spanned by

$$
\xi_{k_{1}, k_{2}}=\partial_{y}-\frac{k_{2}(y)}{4}\left(R_{1}+4 R_{4}\right)+\frac{k_{1}(y)}{2} R_{2}+\frac{1}{2} R_{3},
$$

where the $R_{i}$ form a basis for the Vessiot algebra ${ }^{1}$

$$
\begin{aligned}
& R_{1}=z_{1} \partial_{z_{1}}-z_{2} \partial_{z_{2}}-2 z_{3} \partial_{z_{3}}, \quad R_{2}=\frac{1+z_{1} z_{2}}{z_{1} z_{3}} \partial_{z_{2}}+\partial_{z_{3}}-\frac{z_{4}}{z_{1} z_{2} z_{3}} \partial_{z_{4}}, \\
& R_{3}=z_{1} z_{3} \partial_{z_{1}}-z_{3}^{2} \partial_{z_{3}}, \quad R_{4}=-\frac{1}{4}\left(z_{1} \partial_{z_{1}}-z_{2} \partial_{z_{2}}\right)
\end{aligned}
$$

\footnotetext{
${ }^{1} \mathrm{~A}$ brief geometric construction and interpretation of the Vessiot algebra is given in Section 4 and Appendix A. See [2] for a complete exposition. However, detailed knowledge of Vessiot algebras is not a prerequisite for this paper.
} 
with nonzero structure

$$
\left[R_{1}, R_{2}\right]=2 R_{2}, \quad\left[R_{1}, R_{3}\right]=-2 R_{3}, \quad\left[R_{2}, R_{3}\right]=R_{1} .
$$

Since $\xi_{k_{1}, k_{2}}$ is a curve in the Vessiot algebra it determines an ODE of Lie type. Furthermore $\xi_{k_{1}, k_{2}}$ is generically transverse to the Cauchy data.

Note that $\rho_{1}=R_{1}+4 R_{4}, \rho_{2}=R_{2}, \rho_{3}=R_{3}$ generates a local action of $\operatorname{SL}(2)$ on $\mathbb{R}^{4}$ :

$$
\left[\rho_{1}, \rho_{2}\right]=2 \rho_{2}, \quad\left[\rho_{1}, \rho_{3}\right]=-2 \rho_{3}, \quad\left[\rho_{2}, \rho_{3}\right]=\rho_{1}
$$

and the Cauchy vector is

$$
\xi_{k_{1}, k_{2}}=\partial_{y}-k_{2}(y) \rho_{1}+k_{1}(y) \rho_{2}+\frac{1}{2} \rho_{3} .
$$

In summary, vector field $\xi_{k_{1}, k_{2}}$ flows the 1-dimensional initial data solution curve $\widetilde{\gamma}$ of $\theta_{k_{1}, k_{2}}$ to a 2-dimensional solution. This completes the proof of Theorem 1.

Example 1. As an illustrative example we consider the initial value problem

$$
\begin{aligned}
& u_{x y}+\frac{u_{x} u_{y}}{2\left(1+e^{u / 2+v / 2}\right)}=0, \quad v_{x y}+\frac{v_{x} v_{y}}{2\left(1+e^{u / 2+v / 2}\right)}=0, \\
& u_{\left.\right|_{\gamma}}=v_{\left.\right|_{\gamma}}=0,\left.\quad \frac{\partial u}{\partial \boldsymbol{n}}\right|_{\gamma}=\left.\frac{\partial v}{\partial \boldsymbol{n}}\right|_{\gamma}=\sqrt{2},
\end{aligned}
$$

where $\gamma=(x, x)$. Since $x$ and $y$ are light-cone coordinates $x=(\xi+\tau) / 2, y=(\xi-\tau) / 2$, the curve $\gamma$ corresponds to time $\tau=0$. Thus we have constant initial values at time $\tau=0$. We wish to determine the system of Lie type whose solutions corresponds to the solution of this Cauchy problem. We have $\boldsymbol{n}=2^{-1 / 2}\left(\partial_{x}-\partial_{y}\right)$ and we get

$$
u_{\left.x\right|_{\gamma}}=v_{\left.x\right|_{\gamma}}=1, \quad u_{\left.y\right|_{\gamma}}=v_{\left.y\right|_{\gamma}}=-1 .
$$

So our initial curve in $J^{1}$ is $\widehat{\gamma}(x)=(x, x, 0,0,1,-1,1,-1)=\left(x, y, u, v, u_{x}, u_{y}, v_{x}, v_{y}\right)$. This translates to an initial curve in the adapted coordinates

$$
\left(x, y, z_{1}, z_{2}, z_{3}, z_{4}\right)=(x, x, 1,1,-1,1) .
$$

We extend this to a unique 1-dimensional integral of $\Psi$ and get

$$
\widetilde{\gamma}(x)=\left(x, y, z_{1}, z_{2}, z_{3}, z_{4}, a_{1}, a_{2}, a_{3}, b_{1}, b_{2}, b_{3}\right)=\left(x, x, 1,1,-1,1, \frac{1}{2}, 0,0, \frac{1}{2}, 0,0\right) .
$$

Thus we get

$$
k_{1}(y)=1 / 2, \quad k_{2}(y)=0 .
$$

The Cauchy vector is therefore

$$
\xi_{\frac{1}{2}, 0}=\partial_{y}+\frac{1}{4} R_{2}+\frac{1}{2} R_{3}
$$

We flow this vector field obtaining solutions $z_{i}(x, y)$ subject to the intial conditions

$$
z_{1}(x, x)=1, \quad z_{2}(x, x)=1, \quad z_{3}(x, x)=-1, \quad z_{4}(x, x)=1 .
$$

The ODE are

$$
\frac{\partial z_{1}}{\partial y}=z_{1} z_{2}, \quad \frac{\partial z_{2}}{\partial y}=\frac{1+z_{1} z_{2}}{4 z_{1} z_{3}}, \quad \frac{\partial z_{3}}{\partial y}=\frac{1}{4}-\frac{z_{3}^{2}}{2}, \quad \frac{\partial z_{4}}{\partial y}=\frac{z_{4}}{4 z_{1} z_{2} z_{3}}
$$


to be solved for $z_{i}(x, y)$ subject to the given initial conditions. In fact, we need not solve the whole system but only the equation for $z_{3}$ and then substitute this into the equation for $z_{1}$. This gives the function $u$ up to a quadrature after which the function $v$ can be obtained algebraically from the PDE itself

$$
e^{v / 2}=-e^{-u / 2}\left(1+\frac{u_{x} u_{y}}{2 u_{x y}}\right) .
$$

We get the unique solution of the Cauchy problem to be

$$
u(x, y)=v(x, y)=\ln \left(\cosh \frac{\sqrt{2}}{4}(x-y)+\sqrt{2} \sinh \frac{\sqrt{2}}{4}(x-y)\right)^{2} .
$$

Example 2. A slightly more interesting example is obtained from the initial conditions

$$
u_{\left.\right|_{\gamma}}=2 \ln \lambda, \quad v_{\left.\right|_{\gamma}}=2 \ln \frac{1}{\lambda},\left.\quad \frac{\partial u}{\partial \boldsymbol{n}}\right|_{\gamma}=\left.\frac{\partial v}{\partial \boldsymbol{n}}\right|_{\gamma}=1
$$

for any $\lambda>0$. The Cauchy vector is $\xi_{\frac{1}{4}, 0}$ and its flow subject to the initial conditions along $y=x$ being

$$
z_{1}=\lambda, \quad z_{2}=\frac{1}{\lambda}, \quad z_{3}=-\frac{1}{\sqrt{2}}, \quad z_{4}=\frac{1}{\sqrt{2}}
$$

gives rise to the unique solution

$$
\begin{aligned}
& u=2 \ln \left(\lambda \exp \left(\frac{x+y}{4}\right) \frac{\exp \left(-\frac{y}{2}\right)(3+2 \sqrt{2})-\exp \left(-\frac{x}{2}\right)}{2(1+\sqrt{2})}\right), \\
& v=2 \ln \left(\frac{1}{2 \lambda} \exp \left(\frac{x+y}{4}\right) \frac{\exp (-y)(2 \sqrt{2}+3)-\exp (-x)(2 \sqrt{2}-3)-2 \exp \left(-\frac{x+y}{2}\right)}{\exp \left(-\frac{y}{2}\right)(1+\sqrt{2})+\exp \left(-\frac{x}{2}\right)(1-\sqrt{2})}\right) .
\end{aligned}
$$

Thus, even constant initial data has the potential of producing some interesting explicit solutions. Indeed, one can ask if this solution is global in time.

The fact that we only had to solve for $z_{3}$ in Example 1 (and Example 2) holds not only for these illustrative examples since the system of Lie type in the general case is

$$
\begin{aligned}
& \frac{\partial z_{1}}{\partial y}=\frac{1}{2} z_{1} z_{3}, \quad \frac{\partial z_{2}}{\partial y}=k_{1}(y) \frac{\left(1+z_{1} z_{2}\right)}{2 z_{1} z_{3}} \\
& \frac{\partial z_{3}}{\partial y}=\frac{1}{2}\left(k_{1}(y)+k_{2}(y) z_{3}-z_{3}^{2}\right), \quad \frac{\partial z_{4}}{\partial y}=-\frac{k_{1}(y) z_{4}}{2 z_{1} z_{2} z_{3}} .
\end{aligned}
$$

This proves

Theorem 2. In the Cauchy problem for wave maps

$$
\left(\mathbb{R}^{1,1}, d x d y\right) \rightarrow\left(M, \frac{d u_{1}^{2}-d u_{2}^{2}}{1+e^{-u_{1}}}\right)
$$

of Theorem 1, let functions $k_{1}(y)$ and $k_{2}(y)$ be defined as described above. Denote by $\zeta$ the initial value of $z_{3}=u_{y}$ along $\gamma$. Let $\Gamma$ be the unique function satisfying Riccati initial value problem

$$
\frac{\partial \Gamma}{\partial y}=\frac{1}{2}\left(k_{1}(y)+k_{2}(y) \Gamma-\Gamma^{2}\right), \quad \Gamma_{\left.\right|_{\gamma}}=\zeta .
$$


Define function $u$ to be the unique solution of

$$
\frac{\partial u}{\partial y}=\Gamma, \quad u_{\left.\right|_{\gamma}}=\phi_{1}
$$

and let $v$ be defined algebraically from the partial differential equation

$$
u_{x y}+\frac{u_{x} u_{y}}{2\left(1+e^{u / 2+v / 2}\right)}=0
$$

upon substituting solution $u$ and solving for $v$. The functions $u, v$ constitute the unique solution of the Cauchy problem.

This implies that the solution of any given Cauchy problem for wave maps into the metric

$$
h=\frac{d u_{1}^{2}-d u_{2}^{2}}{1+e^{-u_{1}}}
$$

relies on the solution of a Riccati initial value problem together with one quadrature. The interesting point here is that the Riccati equation is the simplest non-trivial equation of Lie type. It is a Lie system for the local $\mathrm{SL}(2)$-action on the real line that globalises on $\mathbb{R P}^{1}$. In general, solutions of Riccati equations develop singularities in finite time, even those with constant coefficients. However, the theorem above provides a correspondence between Cauchy data for the wave map and the Riccati initial value problem (6). An interesting problem is to study this correspondence and link the nature of the Cauchy data with the properties of the solution of (6) and in turn, link this correspondence with the geometry of the target metric.

For the standard initial value problem where Cauchy data is posed along $0=2 \tau=x-y$, the relationship between the Cauchy data and the coefficients of the Riccati equation is complicated. However, due to its significance and for latter use, we give it explicitly:

$$
\begin{aligned}
k_{1}= & -\frac{1}{4} \frac{\psi_{2}\left(\sqrt{2} \phi_{1 x}-2 \psi_{1}\right) \exp \left(\frac{\phi_{2}}{2}\right)}{\exp \left(-\frac{\phi_{1}}{2}\right)+\exp \left(\frac{\phi_{2}}{2}\right)}, \quad a_{1}=\frac{1}{4} \frac{\left(\sqrt{2} \phi_{1 x}+2 \psi_{1}\right)\left(\sqrt{2} \phi_{2 x}+\psi_{2}\right) \exp \left(\frac{\phi_{1}}{2}\right)}{\exp \left(\frac{\phi_{1}}{2}\right)+\exp \left(-\frac{\phi_{2}}{2}\right)}, \\
k_{2}= & \delta^{-1}\left(2\left(\phi_{1 x}^{2}+2 \psi_{2}^{2}-2 \sqrt{2} \psi_{1} \phi_{1_{x}}-4 k_{1}\right)\left(\psi_{2}+\sqrt{2} \phi_{2 x}\right)\right. \\
& \left.+4 \sqrt{2} a_{1}\left(\phi_{1 x}-\psi_{1} \sqrt{2}\right) \exp \left(-\frac{\phi_{1}}{2}-\frac{\phi_{2}}{2}\right)+8\left(-\psi_{1 x} \sqrt{2}+\phi_{1 x x}\right)\left(\psi_{2}+\sqrt{2} \phi_{2 x}\right)\right),
\end{aligned}
$$

where

$$
\delta=4\left(\sqrt{2} \phi_{2 x}+\psi_{2}\right)\left(\phi_{1_{x}}-\psi_{1} \sqrt{2}\right) .
$$

Recall that functions $\phi_{1}, \phi_{2}$ are respectively the values of $u$ and $v$ along $\gamma$, the $\psi_{i}$ are the values of the normal derivatives along $\gamma$ as stated in the theorem. For instance for arbitrary constant initial conditions along the time axis

$$
\begin{aligned}
& \phi_{1}(x)=u(x, x)=2 \ln \lambda_{1}, \quad \phi_{2}(x)=v(x, x)=2 \ln \lambda_{2}, \\
& \psi_{1}=\alpha \sqrt{2}, \quad \psi_{2}=\beta \sqrt{2} ; \quad \lambda_{1}, \lambda_{2}>0,
\end{aligned}
$$

we have

$$
k_{1}(y)=\frac{\alpha \beta \lambda_{1} \lambda_{2}}{1+\lambda_{1} \lambda_{2}}, \quad k_{2}(y)=-\frac{(\alpha-\beta) \lambda_{1} \lambda_{2}}{1+\lambda_{1} \lambda_{2}} .
$$

The solution of the Cauchy problem in this case is easily calculated as we did in Examples 1 and 2 because it amounts to solving Riccati equation (6) with constant coefficients $k_{1}(y), k_{2}(y)$ given by (7); indeed Example 1 is the choice $\lambda_{1}=\lambda_{2}=1, \alpha=\beta=\sqrt{2}$. However the general formula is complicated and of itself not very informative so we refrain from recording it here. 


\section{Cauchy problem for wave maps and Lie reduction}

We have shown that to solve the Cauchy problem for the wave map equation it is enough to "evolve" the initial data curve $\widetilde{\gamma}$ by solving a Riccati equation which is notably the simplest nontrivial system of Lie type. One significant feature of Lie systems is that they admit "reduction by particular solutions", otherwise known as Lie reduction. There are only a few sources scattered in the literature on this topic, among them [4, 5, 7]. In this section we give an illustration of how Lie reduction may be useful in resolving instances of the Cauchy problem for our wave map system. Appendix A.2 of this paper summarises the known results on systems of Lie type, oriented toward the applications at hand. In this and subsequent sections we will adopt the notation and theory set out in Appendix A.2, to which we refer the reader.

Consider the Cauchy problem for wave maps with (non-constant) Cauchy data

$$
\phi_{1}=\phi_{2}=0, \quad \psi_{1}=-2 \sqrt{2}, \quad \psi_{2}=2 \sqrt{2} x
$$

along the curve $y=x$. We get

$$
k_{1}(y)=-2 y, \quad k_{2}(y)=-y-1,
$$

so that the corresponding Riccati initial value problem of Theorem 2 is

$$
\frac{\partial \Gamma}{\partial y}=\frac{1}{2}\left(-2 y-(y+1) \Gamma-\Gamma^{2}\right), \quad \Gamma(x, x)=2 .
$$

We observe that $\Gamma=1-y$ is a solution vanishing at $y=1$. Implementing the procedure described in Appendix A.2 obtains one factor in the fundamental solution

$$
g_{0}(y)=\left(\begin{array}{cc}
1 & 1-y \\
0 & 1
\end{array}\right) \text {. }
$$

A curve in the isotropy group of 0 is denoted by $H$ and has the form

$$
g_{1}(y)=\left(\begin{array}{cc}
\gamma_{1}(y) & 0 \\
\gamma_{2}(y) & \gamma_{1}(y)^{-1}
\end{array}\right) \text {. }
$$

The curve of Lie algebra elements associated to the Riccati equation

$$
\frac{d z}{d y}=\alpha_{0}(y)+2 \alpha_{1}(y) z-\alpha_{2}(y) z^{2}
$$

is

$$
A(y)=\left(\begin{array}{cc}
\alpha_{1} & \alpha_{0} \\
\alpha_{2} & -\alpha_{1}
\end{array}\right) \subset \mathfrak{s l}(2) .
$$

For the Riccati initial value problem (8) we have

$$
A(y)=\left(\begin{array}{cc}
-\frac{y+1}{4} & y \\
\frac{1}{2} & \frac{y+1}{4}
\end{array}\right)
$$

and the reduced fundamental equation is

$$
\frac{d g_{1}}{d y}=B(y) g_{1},
$$


where (see Appendix A.2, especially Theorem 6 \& Appendix A.2.1)

$$
B(y)=g_{0}(y)^{-1} A(y) g_{0}(y)-g_{0}(y)^{-1} \frac{d g_{0}}{d y}=\left(\begin{array}{cc}
\frac{1}{4} y-\frac{3}{4} & 0 \\
\frac{1}{2} & -\frac{1}{4} y+\frac{3}{4}
\end{array}\right),
$$

valued in the isotropy subalgebra at 0 , as expected. The ODE initial value problem for the fundamental solution factor $g_{1}(y)$ is

$$
\frac{d \gamma_{1}}{d y}-\beta_{1} \gamma_{1}=0, \quad \frac{d \gamma_{2}}{d y}-\frac{1}{2} \gamma_{1}+\beta_{1} \gamma_{2}=0, \quad \gamma_{1}(1)=1, \quad \gamma_{2}(1)=0,
$$

where $\beta_{1}=(y-3) / 4$. This problem can be explicitly solved in terms of elementary functions giving

$$
\begin{aligned}
& \gamma_{1}(y)=\exp \left(\frac{(y-1)(y-5)}{8}\right) \\
& \gamma_{2}(y)=\sqrt{\pi} \exp \left(\frac{3}{4} y-y^{2}-\frac{13}{8}\right)\left(\operatorname{erfi}(1)+\operatorname{erfi}\left(\frac{1}{2}(y-3)\right)\right),
\end{aligned}
$$

where $i=\sqrt{-1}$ and erfi denotes a concomitant of the error function:

$$
\operatorname{erfi}(x)=\frac{2}{\sqrt{\pi}} \int_{0}^{x} \exp \left(t^{2}\right) d t
$$

This data enables us to construct the fundamental solution $g(y)=g_{0}(y) g_{1}(y)$ for (8) and leads to the solution of the ODE in (8)

$$
\Gamma(y ; q)=\lambda_{g(y)}(q),
$$

where $\lambda_{h}$ denotes the linear fractional transformation (12) by $h \in \mathrm{SL}(2)$.

It is now a simple matter to determine the value of $q$ that satisfies the initial condition $\Gamma(x, x)=2$ and giving the unique solution $\Gamma(x, y)$ of the Riccati initial value problem (8). We find

$$
\Gamma(x, y)=1-y-\frac{2 \exp \left(\frac{3}{2} y-\frac{1}{4} y^{2}\right)}{\Delta(x)-\sqrt{\pi} \exp \left(-\frac{9}{4}\right) \operatorname{erfi}\left(\frac{1}{2}(y-3)\right)},
$$

where

$$
\Delta(x)=-\frac{2 \exp \left(-\frac{3}{2} x+\frac{1}{4} x^{2}\right)}{(1+x)}+\sqrt{\pi} \exp \left(-\frac{9}{4}\right) \operatorname{erfi}\left(\frac{1}{2}(x-3)\right) .
$$

Finally, we obtain an integral representation of the solution $u$ satisfying

$$
\frac{\partial u}{\partial y}=\Gamma(x, y), \quad u(x, x)=0,
$$

namely

$$
u(x, y)=\int_{x}^{y} \Gamma(x, s) d s
$$

or in terms of spacetime coordinates $(\xi, \tau)$

$$
\bar{u}(\xi, \tau)=\int_{\frac{1}{2}(\xi+\tau)}^{\frac{1}{2}(\xi-\tau)} \Gamma\left(\frac{1}{2}(\xi+\tau), s\right) d s .
$$


The significance of Lie reduction in our ability to solve the Riccati equation should here be emphasised. Without this, it would have been impossible to construct the fundamental solution and there would be no hope of constructing function $\Gamma$ and constructing the integral representation of the solution of a Cauchy problem with non-constant initial data.

In the example above we relied on knowledge of a simple solution, namely $\Gamma(x, y)=1-y$ to perform the reduction. But even with polynomial or rational coefficients a Riccati equation will not generally have any rational solutions. In this case however we can appeal to the well known fact that every Riccati equation can be linearised.

Lemma 1. The general Riccati equation

$$
\frac{d z}{d t}=\alpha_{0}(t)+2 \alpha_{1}(t) z(t)-\alpha_{2}(t) z(t)^{2}
$$

can be transformed to the form

$$
\frac{d}{d \tau} y(\tau)=\beta(\tau)+y(\tau)^{2}
$$

where $z=p(t) y(t)$

$$
\ln p=\int^{t} 2 \alpha_{1}(s) d s, \quad \tau=\int^{t} \alpha_{2}(s) p(s) d s .
$$

Provided these quadratures can be carried out then the explicit solvability of (9) depends on the properties of its linearisation

$$
\frac{d^{2} \psi}{d \tau^{2}}+\beta(\tau) \psi=0, \quad \text { where } \quad y(\tau)=-\frac{1}{\psi(\tau)} \frac{d}{d \tau} \psi(\tau)
$$

Any solution of the 2 nd order linear ODE can be used in the Lie reduction of the Riccati equation.

As a consequence of Lemma 1 and Theorem 7 of Appendix A.2, we have

Corollary 1. The solution of the Cauchy problem for wave maps of Theorem 1

$$
\left(\mathbb{R}^{1,1}, d x d y\right) \rightarrow\left(M, \frac{d u_{1}^{2}-d u_{2}^{2}}{1+e^{-u_{1}}}\right)
$$

is reducible to quadrature provided a particular solution of the Riccati equation (6) is known. A particular solution of (6) can be constructed by quadrature and the solution of a linear second order ODE.

Remark 1. As a consequence of Lemma 1 and Theorem 7 the differential equations solver in MAPLE - dsolve is very often able to construct an explicit representation in terms of known special functions to a Riccati initial value problem when the coefficients are polynomial functions of the independent variable.

\section{Hyperbolic Weierstrass representation}

In this section we use the Darboux integrability of the wave map equation (2) to compute its general solution and hence construct a hyperbolic Weierstrass-type representation for wave maps into the corresponding metric. According to [2], we pull back $\Psi$ to a suitable integral manifold 
$M_{1}, M_{2}$ of $H_{1}^{(\infty)}$ and $H_{2}^{(\infty)}$ respectively. It is convenient to define $M_{1}$ by $y=b_{1}=b_{2}=b_{3}=0$ and $M_{2}$ by $x=a_{1}=a_{2}=a_{3}=0$. This gives Pfaffian systems

$$
\begin{aligned}
\Psi_{1}=\left\{d z_{4}-\frac{1}{2}\left(a_{3} z_{4}+a_{1}-z_{4}^{2}\right) d x, d z_{3}+\frac{1}{2 z_{1} z_{2} z_{4}} a_{1} z_{3} d x, d z_{2}-\frac{1}{2} z_{2} z_{4} d x,\right. \\
\left.d z_{1}-\frac{1}{2 z_{2} z_{4}} a_{1}\left(1+z_{1} z_{2}\right) d x, d a_{1}-a_{2} d x\right\}
\end{aligned}
$$

and

$$
\begin{aligned}
\Psi_{2}=\left\{d z_{1}-\right. & \frac{1}{2} z_{3} z_{1} d y, d z_{2}-\frac{1}{2 z_{1} z_{3}} b_{1}\left(1+z_{1} z_{2}\right) d y, d z_{4}+\frac{1}{2 z_{1} z_{2} z_{3}} b_{1} z_{4} d y \\
& \left.d z_{3}-\frac{1}{2}\left(b_{3} z_{3}-z_{3}^{2}+b_{1}\right) d y, d b_{1}-b_{2} d y\right\}
\end{aligned}
$$

each of rank 5 on 8-manifolds: $\left(M_{i}, \Psi_{i}\right)$. Locally $M_{1}$ has coordinates $x, z_{1}, z_{1}, z_{2}, z_{3}, z_{4}, a_{1}, a_{2}$, $a_{3}$ while $M_{2}$ has local coordinates $y, z_{1}, z_{1}, z_{2}, z_{3}, z_{4}, b_{1}, b_{2}, b_{3}$. Using these local formulas, we define a local product structure

$$
\left(\widehat{M}_{1} \times \widehat{M}_{2}, \widehat{\Psi}_{1} \oplus \widehat{\Psi}_{2}\right)
$$

where

$$
\begin{aligned}
\widehat{\Psi}_{1}=\left\{d q_{4}-\right. & \frac{1}{2}\left(a_{3} q_{4}+a_{1}-q_{4}^{2}\right) d x, d q_{3}+\frac{1}{2 q_{1} q_{2} q_{4}} a_{1} q_{3} d x, d q_{2}-\frac{1}{2} q_{2} q_{4} d x \\
& \left.d q_{1}-\frac{1}{2 q_{2} q_{4}} a_{1}\left(1+q_{1} q_{2}\right) d x, d a_{1}-a_{2} d x\right\}
\end{aligned}
$$

and

$$
\begin{aligned}
\widehat{\Psi}_{2}=\{ & d p_{1}-\frac{1}{2} p_{1} p_{3} d y, d p_{2}-\frac{1}{2 p_{1} p_{3}} b_{1}\left(1+p_{1} p_{2}\right) d y, d p_{4}+\frac{1}{2 p_{1} p_{2} p_{3}} b_{1} p_{4} d y \\
& \left.d p_{3}-\frac{1}{2}\left(b_{3} p_{3}-p_{3}^{2}+b_{1}\right) d y, d b_{1}-b_{2} d y\right\}
\end{aligned}
$$

As described in [2], the relationship between $\Psi, \widehat{\Psi}_{1}$ and $\widehat{\Psi}_{2}$ is that every integral manifold of $\Psi$ is a superposition of an integral manifold of $\widehat{\Psi}_{1}$ and $\widehat{\Psi}_{2}$. The superposition formula is the map $^{2}$

$$
\boldsymbol{\sigma}: \widehat{M}_{1} \times \widehat{M}_{2} \rightarrow M
$$

defined by

$$
\begin{gathered}
\boldsymbol{\sigma}(x, \boldsymbol{p}, \boldsymbol{a} ; y, \boldsymbol{q}, \boldsymbol{b})=\left(\frac{\left.1-p_{1} p_{2} p_{4}+2 p_{1} p_{2} p_{4} q_{3}-p_{1} p_{2} q_{3}-q_{3}+p_{1} p_{2}\right) q_{1}}{p_{4} p_{2}},\right. \\
\frac{p_{2}\left(2 p_{4} q_{3} q_{2} q_{1}-q_{1} q_{2} q_{3}-p_{4} q_{2} q_{1}+q_{1} q_{2}-p_{4}+1\right)}{q_{3} q_{1}}, \\
\frac{\left(2 p_{4} q_{3}-q_{3}-p_{4}+1\right) p_{3} p_{1} p_{2}}{1-p_{1} p_{2} p_{4}+2 p_{1} p_{2} p_{4} q_{3}-p_{1} p_{2} q_{3}-q_{3}+p_{1} p_{2}},
\end{gathered}
$$

\footnotetext{
${ }^{2}$ See Appendix A for further details on the superposition formula; in particular, how it is defined and constructed.
} 


$$
\begin{aligned}
& \left.\frac{\left(2 p_{4} q_{3}-q_{3}-p_{4}+1\right) q_{1} q_{2} q_{4}}{2 p_{4} q_{3} q_{2} q_{1}-q_{1} q_{2} q_{3}-p_{4} q_{2} q_{1}+q_{1} q_{2}-p_{4}+1}, x, y, a_{1}, a_{2}, a_{3}, b_{1}, b_{2}, b_{3}\right) \\
= & \left(z_{1}, z_{2}, z_{3}, z_{4}, x, y, a_{1}, a_{2}, a_{3}, b_{1}, b_{2}, b_{3}\right) .
\end{aligned}
$$

The usefulness of this factorisation of the integration problem for $\Psi$ is not only that the integration of $\Psi_{i}$ relies on ODE while that of $\Psi$ relies on PDE but that the $\Psi_{i}$ are locally equivalent to prolongations of the contact system on $J^{1}\left(\mathbb{R}, \mathbb{R}^{2}\right)$. To see this we turn to the characterisation of partial prolongations of such contact systems provided by $[18,19]$ which also provide simple procedures for finding the equivalence. To implement this we compute the annihilators

$$
\begin{aligned}
& \text { ann } \widehat{\Psi}_{1}:=\widehat{H}_{1} \\
& =\left\{\partial_{x}+a_{2} \partial_{a_{1}}+\frac{a_{1}\left(1+q_{1} q_{2}\right)}{2 q_{2} q_{4}} \partial_{q_{1}}+\frac{q_{2} q_{4}}{2} \partial_{q_{2}}-\frac{a_{1} q_{3}}{2 q_{1} q_{2} q_{4}} \partial_{q_{3}}+\frac{1}{2}\left(a_{3} q_{4}+a_{1}-q_{4}^{2}\right) \partial_{q_{4}}, \partial_{a_{2}}, \partial_{a_{3}}\right\}, \\
& \text { ann } \widehat{\Psi}_{2}:=\widehat{H}_{2} \\
& =\left\{\partial_{y}+b_{2} \partial_{b_{1}}+\frac{p_{1} p_{3}}{2} \partial_{p_{1}}+\frac{b_{1}\left(1+p_{1} p_{2}\right)}{2 p_{1} p_{3}} \partial_{p_{2}}+\frac{1}{2}\left(b_{3} p_{3}-p_{3}^{2}+b_{1}\right) \partial_{p_{3}}-\frac{b_{1} p_{4}}{2 p_{1} p_{2} p_{3}} \partial_{p_{4}}, \partial_{b_{2}}, \partial_{b_{3}}\right\} .
\end{aligned}
$$

We show that each of the $\widehat{H}_{i}$ is locally equivalent to the partial prolongation $C\langle 0,1,1\rangle$ of the contact distribution on $J^{1}\left(\mathbb{R}, \mathbb{R}^{2}\right)$. That is, the contact distribution on $J^{1}\left(\mathbb{R}, \mathbb{R}^{2}\right)$ partially prolonged so that one dependent variable has order 2 and the other order 3 with canonical local normal form

$$
C\langle 0,1,1\rangle=\left\{\partial_{t}+z_{1}^{1} \partial_{z^{1}}+z_{2}^{1} \partial_{z_{1}^{1}}+z_{1}^{2} \partial_{z^{2}}+z_{2}^{2} \partial_{z_{1}^{2}}+z_{3}^{2} \partial_{z_{2}^{2}}, \partial_{z_{2}^{1}}, \partial_{z_{3}^{2}}\right\} .
$$

Let $\mathcal{D}$ be a smooth distribution on manifold $M$ and assume that $\mathcal{D}$ is totally regular in the sense that $\mathcal{D}$, all its derived bundles and all their corresponding Cauchy bundles have constant rank on $M$. Denote $m_{i}=\operatorname{dim} \mathcal{D}^{(i)}, \chi^{i}=\operatorname{dim} \operatorname{Char} \mathcal{D}^{(i)}$ and $\chi_{i-1}^{i}=\operatorname{dim}$ Char $\mathcal{D}_{i-1}^{(i)}$, where

$$
\text { Char } \mathcal{D}_{i-1}^{(i)}=\mathcal{D}^{(i-1)} \cap \operatorname{Char} \mathcal{D}^{(i)} \text {. }
$$

Below, $k$ denotes the derived length of $\mathcal{D}$.

According to Theorem 4.1 of [18], a totally regular distribution $\mathcal{D}$ on smooth manifold $M$ is locally equivalent to a partial prolongation of the contact distribution on $J^{1}\left(\mathbb{R}, \mathbb{R}^{q}\right)$ for some $q$ if and only if

1. The integers $m_{i}, \chi^{j}, \chi_{j-1}^{j}$ satisfy the numerical constraints

$$
\chi^{j}=2 m_{j}-m_{j+1}-1, \quad 0 \leq j \leq k-1, \quad \chi_{i-1}^{i}=m_{i}-1, \quad 1 \leq i \leq k-1 .
$$

2. If $m_{k}-m_{k-1}>1$ then a certain canonically associated bundle called the resolvent is integrable ${ }^{3}$.

A pair $(M, \mathcal{D})$ that satisfies these conditions is said to be a Goursat manifold or Goursat bundle. Moreover, if $\mathcal{D}$ is a Goursat bundle on $M$ then it is locally equivalent to a partial prolongation with $\chi^{j}-\chi_{j-1}^{j}$ dependent variables at order $j<k$ and $m_{k}-m_{k-1}$ dependent variables at highest order, $k$. This uniquely identifies the partial prolongation associated to a given Goursat manifold. Before discussing the question of constructing equivalences, let us

\footnotetext{
${ }^{3}$ In the original formulation of Theorem 4.1 in [18], the integrability of Char $\mathcal{D}_{i-1}^{(i)}$ is an additional hypothesis to be checked. This is a simple task but unnecessary since it is easy to see that this bundle is always integrable and the hypothesis can be omitted.
} 
solve the recognition problem for the distributions $\widehat{H}_{i}$. We take $\widehat{H}_{1}$ which we denote temporarily by $\widehat{K}$. We find

$$
\begin{aligned}
& \text { Char } \widehat{K}=\{0\}, \quad \text { Char } \widehat{K}^{(1)}=\left\{\partial_{a_{2}}, \partial_{a_{3}}\right\}, \quad \text { Char } \widehat{K}_{1}^{(2)}=\left\{\partial_{a_{2}}, \partial_{a_{3}}, \partial_{a_{1}}, \partial_{q_{3}}\right\}, \\
& \text { Char } \widehat{K}^{(2)}=\left\{\partial_{a_{1}}, \partial_{a_{2}}, \partial_{a_{3}}, \partial_{q_{3}}, \partial_{x}\right\} .
\end{aligned}
$$

Calculation shows that the dimensions of the derived bundles are

$$
\operatorname{dim} \widehat{K}=3, \quad \operatorname{dim} \widehat{K}^{(1)}=5, \quad \operatorname{dim} \widehat{K}^{(2)}=7, \quad \operatorname{dim} \widehat{K}^{(s)}=8, \quad s \geq 3 .
$$

Hence the derived length is $k=3$. Below we check the first condition of a Goursat bundle.

Table. Checking the numerical constraints satisfied by $(M, \mathcal{D})$.

\begin{tabular}{|c|c|c|c|c|c|}
\hline$j$ & $m_{j}$ & $m_{j-1}-1$ & $2 m_{j}-m_{j+1}-1$ & $\chi_{j-1}^{j}$ & $\chi^{j}$ \\
\hline 0 & 3 & - & $6-5-1=0$ & - & 0 \\
1 & 5 & 2 & $10-7-1=2$ & 2 & 2 \\
2 & 7 & 4 & $14-8-1=5$ & 4 & 5 \\
\hline
\end{tabular}

Hence, $\widehat{K}$ is a Goursat bundle with $k=3, m_{k}-m_{k-1}=1$ and the only nonzero difference $\chi^{j}-\chi_{j-1}^{j}$ being at order $j=2: \chi^{2}-\chi_{1}^{2}=5-4=1$. Hence there is one variable of order 2 and one variable of order 3 . This solves the recognition problem and we can assert that $\widehat{K}:=\widehat{H}_{1}$ is locally equivalent to $C\langle 0,1,1\rangle$. Next, we show how to construct an equivalence. Given a Goursat bundle, an efficient method for constructing an equivalence map was worked out in [19] and relies on the filtration induced on the cotangent bundle. Denote by $\Xi^{(j)}$ and $\Xi_{j-1}^{(j)}$ the annihilators of Char $\widehat{K}^{(j)}$ and Char $\widehat{K}_{j-1}^{(j)}$, respectively. Then we obtain the filtration

$$
\Xi^{(2)} \subset \Xi_{1}^{(2)} \subset \Xi^{(1)}
$$

spanned as

$$
\left\{d q_{1}, d q_{2}, d q_{3}\right\} \subset\left\{d q_{1}, d q_{2}, d q_{3}, d x\right\} \subset\left\{d q_{1}, d q_{2}, d q_{3}, d x, d q_{4}, d a_{1}\right\} .
$$

The construction proceeds by building appropriate differential operators and functions. Because $m_{k}-m_{k-1}=1$, condition 2 of the definition of Goursat manifold is vacuous. Instead we fix any first integral of Char $\mathcal{D}^{(k-1)}$, denoted, $t$ and any section $Z$ of $\mathcal{D}$ such that $Z t=1$. Then, define a distribution $\Pi^{k}$ inductively as follows:

$$
\Pi^{\ell+1}=\left[Z, \Pi^{\ell}\right], \quad \Pi^{1}=\text { Char } \mathcal{D}_{0}^{1}, \quad 1 \leq \ell \leq k-1 .
$$

There is a function $\varphi^{k}$ which is a first integral of $\Pi^{k}$ such that $d \varphi^{k} \wedge d t \neq 0$. The function $\varphi^{k}$ is said to be a fundamental function of order $k$. The space of fundamental functions of lower order can be constructed from the filtration above by taking quotients. Specifically, as noted above, in this case the only fundamental functions of less than maximal order 3 are of order 2 . They are described by the quotient bundle

$$
\Xi_{1}^{(2)} / \Xi^{(2)}=\{d x\} \quad \bmod d q_{1}, d q_{2}, d q_{3} .
$$

Without loss of generality we can take $\varphi^{2}=x$ to be a fundamental function of order 2 . The construction of the equivalence map is now as follows. Function $t$ is the "independent variable" and successive differentiation gives the higher order variables

$$
\begin{aligned}
& z^{1}=\varphi^{2}, \quad z_{1}^{1}=Z \varphi^{2}, \quad z_{2}^{1}=Z^{2} \varphi^{2}, \\
& z^{2}=\varphi^{k}, \quad z_{1}^{2}=Z \varphi^{k}, \quad z_{2}^{2}=Z^{2} \varphi^{k}, \quad z_{3}^{2}=Z^{3} \varphi^{k} .
\end{aligned}
$$


We now implement this. The first integrals of Char $\widehat{K}^{(2)}$ are spanned by $q_{1}, q_{2}, q_{3}$ and any function of these can be chosen to be $t$. If we choose (say) $t=q_{2}$, then for $Z$ we choose

$$
Z=\frac{2}{q_{2} q_{4}} X
$$

where $X$ is the first vector field in the basis for $\widehat{K}$ above; for then $Z t=1$, as required. We then construct the integrable distribution $\Pi^{3}$ as described above and discover that its first integrals are spanned by

$$
q_{2} \quad \text { and } \quad \frac{q_{1} q_{3}}{1+q_{1} q_{2}} .
$$

Hence the fundamental function of highest order is

$$
z^{2}=\varphi^{k}=\frac{q_{1} q_{3}}{1+q_{1} q_{2}} .
$$

The data

$$
t=q_{2}, \quad z^{1}=x, \quad z^{2}=\frac{q_{1} q_{3}}{1+q_{1} q_{2}}
$$

and differentiation by $Z$ now constructs the local equivalence $\psi$ identifying $\widehat{K}=\widehat{H}_{1}$ and $C\langle 0,1,1\rangle$. The local inverse $\psi_{1}^{-1}: \mathbb{R} \rightarrow \widehat{M}_{1}$ determines the integral submanifolds of $\widehat{H}_{1}$.

An exactly analogous calculation holds for $\widehat{H}_{2}$ and one arrives thereby at an explicit map $\psi_{2}^{-1}: \mathbb{R} \rightarrow \widehat{M}_{2}$ representing the integral manifolds of $\widehat{H}_{2}$. The explicit integral manifolds of $\Psi$ are a superposition of those of $\widehat{H}_{1}$ and $\widehat{H}_{2}$ :

$$
\mathbb{R} \times \mathbb{R} \rightarrow \boldsymbol{\sigma}\left(\psi_{1}^{-1}(\mathbb{R}), \psi_{2}^{-1}(\mathbb{R})\right) .
$$

In this way we obtain remarkably compact representations for wave maps into this metric:

Theorem 3 (hyperbolic Weierstrass representation). For each collection of twice continuously differentiable real valued functions $f_{1}(s), f_{2}(s), g_{1}(t), g_{2}(t)$ of parameters $s, t$, the functions

$$
\begin{aligned}
& x=f_{1}(s), \quad y=g_{1}(t), \\
& e^{u / 2}=\frac{\left(t g_{2}(t)-1\right) \dot{f}_{2}(s)+\left(2 t g_{2}(t)-1\right) f_{2}(s)^{2}}{g_{2}(t)\left(f_{2}(s)+s \dot{f}_{2}(s)\right)}, \\
& e^{v / 2}=\frac{\left(s f_{2}(s)-1\right) \dot{g}_{2}(t)+\left(2 s f_{2}(s)-1\right) g_{2}(t)^{2}}{f_{2}(s)\left(g_{2}(t)+t \dot{g}_{2}(t)\right)}
\end{aligned}
$$

define harmonic maps

$$
\left(\mathbb{R}^{1,1}, d x d y\right) \rightarrow\left(N, \frac{d u_{1}^{2}-d u_{2}^{2}}{1+e^{-u_{1}}}\right) \quad \text { by } \quad u_{1}=u+v, \quad u_{2}=u-v .
$$

\section{Concluding remarks}

We've seen that for a large family of non-constant initial data, it is possible to construct explicit integral representations for solutions of the Cauchy problem for wave maps into a certain nonconstant curvature metric due to the fact that the corresponding Euler-Lagrange equation is Darboux integrable and because the Cauchy data can be extended as a flow by a system of Lie type. We have also constructed a hyperbolic Weierstrass representation for such wave maps making use of the general theory in $[2,18,19]$. The fundamental ingredients throughout include 
the theory of systems of Lie type and the notion of a Vessiot group associated to any Darboux integrable exterior differential system [2]. We expressed the evolution of the Cauchy data as a system of Lie type for the action of a subgroup of the Vessiot group.

We may perhaps regret the occurence of integral representations in our solution of the Cauchy problem preferring the elimination of all quadrature. Alas, this is surely a forlorn hope in a difficult nonlinear problem, especially when it is recalled that even in the general Cauchy problem for the (1+1)-linear wave equation quadrature cannot be eliminated, according to the d'Alembert formula. However, one can ask if there are Darboux integrable nonlinear sigma models with solvable Vessiot groups for harmonic maps into nonzero curvature metrics. This would make the application of the theory of systems of Lie type very useful indeed. In fact there is at least one such sigma model [6].

Interesting open problems include: what intrinsic properties of a metric render the corresponding wave map system Darboux integrable? Moreover, what do we learn about the geometry and topology of target manifolds from so vast a reduction in the Cauchy problem? This is especially intriging when it is recalled that the solution to the Cauchy problem has been expressed as a curve or "evolution" in a finite Lie group, arising by Lie's theory from a corresponding curve in its Lie algebra.

\section{A Appendix}

\section{A.1 The superposition formula}

For completeness, in this appendix we make a remark on the construction of the superposition formula $\sigma: \widehat{M}_{1} \times \widehat{M}_{2} \rightarrow M$. A general construction valid for any decomposable exterior differential system was worked out in [2]. Below we present results for the wave map system studied in this paper. The hyperbolic structure of the wave map system in adapted coordinates is given by

$$
H=H_{1} \oplus H_{2},
$$

where

$$
\begin{aligned}
H_{1}= & \left\{\partial_{x}+a_{2} \partial_{a_{1}}+\frac{a_{1}\left(1+z_{1} z_{2}\right)}{2 z_{2} z_{4}} \partial_{z_{1}}+\frac{z_{2} z_{4}}{2} \partial_{z_{2}}-\frac{a_{1} z_{3}}{2 z_{1} z_{2} z_{4}} \partial_{z_{3}}+\frac{1}{2}\left(a_{3} z_{4}+a_{1}-z_{4}^{2}\right) \partial_{z_{4}},\right. \\
& \left.\partial_{a_{2}}, \partial_{a_{3}}\right\}, \\
H_{2}= & \left\{\partial_{y}+b_{2} \partial_{b_{1}}+\frac{z_{1} z_{3}}{2} \partial_{z_{1}}+\frac{b_{1}\left(1+z_{1} z_{2}\right)}{2 z_{1} z_{3}} \partial_{z_{2}}+\frac{1}{2}\left(b_{3} z_{3}-z_{3}^{2}+b_{1}\right) \partial_{z_{3}}-\frac{b_{1} z_{4}}{2 z_{1} z_{2} z_{3}} \partial_{z_{4}},\right. \\
& \left.\partial_{b_{2}}, \partial_{b_{3}}\right\} .
\end{aligned}
$$

Calculation shows that the infinitesimal symmetries of $H_{1}$ which are tangent to the level sets of all the first integrals $x, y, \boldsymbol{a}, \boldsymbol{b}$, the tangential characteristic symmetries of $H_{1}$, are spanned by

$$
\begin{aligned}
\mathcal{E}_{1}=\{ & \frac{1}{2}\left(z_{1} z_{3} \partial_{z_{1}}-z_{3}^{2} \partial_{z_{3}}\right), \frac{1+z_{1} z_{2}}{z_{1} z_{3}} \partial_{z_{2}}+\partial_{z_{3}}-\frac{z_{4}}{z_{1} z_{2} z_{4}} \partial_{z_{4}},-\frac{1}{2}\left(z_{1} \partial_{z_{1}}+z_{2} \partial_{z_{2}}\right)+z_{3} \partial_{z_{3}}, \\
& \left.-\frac{1}{4}\left(z_{1} \partial_{z_{1}}+z_{2} \partial_{z_{2}}\right)\right\} .
\end{aligned}
$$


Similarly, the tangential characteristic symmetries of $H_{2}$ are spanned by

$$
\begin{aligned}
\mathcal{E}_{2}= & \frac{1+z_{1} z_{2}}{2 z_{2} z_{4}} \partial_{z_{1}}-\frac{z_{3}}{z_{1} z_{2} z_{4}} \partial_{z_{3}}+\frac{1}{2} \partial_{z_{4}}, \\
& \left.z_{2} z_{4} \partial_{z_{2}}-z_{4}^{2} \partial_{z_{4}}, \frac{1}{2}\left(z_{1} \partial_{z_{1}}-z_{2} \partial_{z_{2}}\right)+z_{4} \partial_{z_{4}},-\frac{1}{2}\left(z_{1} \partial_{z_{1}}-z_{2} \partial_{z_{2}}\right)\right\} .
\end{aligned}
$$

The structure of these Lie algebras are in reciprocal relation, namely, for $\mathcal{E}_{1}$ the nonzero Lie brackets are

$$
\left[e_{1}, e_{2}\right]=e_{3}, \quad\left[e_{1}, e_{3}\right]=e_{1}, \quad\left[e_{2}, e_{3}\right]=-e_{2},
$$

while for $\mathcal{E}_{2}$ we have

$$
\left[f_{1}, f_{2}\right]=-f_{3}, \quad\left[f_{1}, f_{3}\right]=-f_{1}, \quad\left[f_{2}, f_{3}\right]=f_{2} \quad \text { and } \quad\left[e_{i}, f_{j}\right]=0, \quad \forall i, j .
$$

Hence, $\mathcal{E}_{1}, \mathcal{E}_{2}$ define the isomorphism class of the Vessiot algebra for this Darboux integrable system, in the terminology of [2]. Each $\mathcal{E}_{i}$ is isomorphic to $\mathfrak{s l}(2) \oplus \mathbb{R}$ and each frames a neighbourhood of a point of $\mathbb{R}^{4}$. By the converse of Lie's second fundamental theorem there is a local Lie group $(G, m)$, where $m: G \times G \rightarrow G$ denotes group composition such that $\mathcal{E}_{i}$ coincide with the infinitesimal left and right translations on $G$. From the expressions for either $\mathcal{E}_{1}$ or $\mathcal{E}_{2}$, by computing flows or otherwise, we can compute function $m$. The components of $m$ coincide precisely with the first 4 components of the superposition map $\boldsymbol{\sigma}$, where we interpret $\left(\left\{p_{i}\right\}_{i=1}^{4},\left\{q_{i}\right\}_{i=1}^{4}\right)$ as local coordinates around $(e, e) \in G \times G$; $e$ being the identity in $G$.

Remark 2. The tangential characteristic symmetries [20, 21] described above do not in general lead directly to the Vessiot algebra, which is needed for the construction of the superposition formula. In general a sequence of coframe adaptations described in [2] is required. In terms of these the distribution $H_{1} \oplus H_{2}$ above can be constructed from components of the fifth adapted coframe of a so-called Darboux pair. However, it turns out that for relatively low dimensional examples like the one in this paper, the full machinery of [2] can sometimes be avoided and instead it is convenient to carry out a direct computation of the tangential characteristic symmetries of $H_{1}$ and $H_{2}$ as we did above. A simple example where this procedure is already not sufficient is provided by a Fermi-Pasta-Ulam equation (see [20]), $u_{t t}=u_{x}^{-4} u_{x x}$.

\section{A.2 Systems of Lie type}

Let $\mu: G \times M \rightarrow M$ be a left-action of a Lie group $G$ on manifold $M$ and $\mathcal{G} \subset \mathfrak{X}(M)$ its Lie algebra of infinitesimal generators; $\mathfrak{X}(M)$ is the Lie algebra of all smooth vector fields on $M$. Let $X_{i}$ be a basis for $\mathcal{G}$. Then, a vector field of Lie type or Lie field is a curve in $\mathcal{G}$

$$
\mathcal{X}=\sum_{i} \alpha_{i}(t) X_{i}
$$

where $\alpha_{i}$ are smooth functions of parameter $t$. An ODE of Lie type is the differential equation determined by a Lie field

$$
\frac{d x}{d t}=\mathcal{X}_{\left.\right|_{x(t)}} .
$$

We pause to recall the map $\widehat{\boldsymbol{\mu}}: \mathfrak{g} \rightarrow \mathfrak{X}(M)$, defined by

$$
\widehat{\boldsymbol{\mu}}(u)_{\left.\right|_{x}}=\frac{d}{d \epsilon} \mu\left(\varphi_{\epsilon}(u), x\right)_{\left.\right|_{\epsilon=0}}, \quad \forall u \in \mathfrak{g},
$$


where $\varphi_{\epsilon}(u)$ is the flow (in $G$ ) of $u \in \mathfrak{g}$ and $\mathfrak{g}$ is the matrix Lie algebra associated to $G$, viewed as a matrix group. The map $\widehat{\boldsymbol{\mu}}$ is the standard anti-homomorphism, induced by the left-action $\mu$, from the matrix Lie algebra $\mathfrak{g}$ to the Lie algebra of smooth vector fields $\mathfrak{X}(M)$ on $M$. Remark that for each $x \in M$, ker $\widehat{\boldsymbol{\mu}}_{\left.\right|_{x}}$ is equal to the subalgebra $\mathcal{I}_{x} \subseteq \mathcal{G}$ of infinitesimal generators which vanish at $x$; that is, $\operatorname{ker} \widehat{\boldsymbol{\mu}}_{\mid x}$ is the isotropy subalgebra at $x$. An action $\mu$ is effective on $M$ if the global isotopy group $\mathcal{I}(M)$ is trivial. Recall that $\mathcal{I}(M)$ is the set of all $g \in G$ such that $\mu(g, x)=x$ for all $x \in M$. It follows that if the action is effective then ker $\widehat{\boldsymbol{\mu}}$ is trivial and the map $\widehat{\boldsymbol{\mu}}$ is injective. Henceforth we assume that the $G$-action is effective or at least almost effective; an action is almost effective, if $\mathcal{I}(M)$ is discrete. In this case, $\widehat{\boldsymbol{\mu}}$ is injective for those elements of $\mathfrak{g}$ whose flows are close to the the identity element in $G$.

We will also assume that the action is transitive so that for each $x, y \in M$ the corresponding isotropy subalgebras are isomorphic, $\mathcal{I}_{x} \simeq \mathcal{I}_{y}$. We therefore speak of the isotropy subalgebra or subgroup. We now recall some useful notation from [7].

Let $f: M \rightarrow G$ be a smooth map and let $\omega=\delta(f)=d f \cdot f^{-1}$. We know that $\omega$ is a rightinvariant Maurer-Cartan form and that it is valued in the Lie algebra $\mathfrak{g}$ of $G$.

Definition 3. Let $M$ be a smooth manifold and $G$ a Lie group.

1. A differential form $\omega \in \Omega(M, \mathfrak{g})$ is said to be a Maurer-Cartan form if it satisfies

$$
d \omega+\omega \wedge \omega=0 .
$$

2. A map $f: M \rightarrow G$ is said to be an integral of a Maurer-Cartan form $\omega$ if and only if $\delta(f)=\omega$.

Theorem 4. On a simply connected manifold every Maurer-Cartan form has an integral.

We denote the set of all $\mathfrak{g}$-valued Maurer-Cartan forms on $M$ by $\Omega(M, \mathfrak{g})$. Specialising to the case $M=\mathbb{R}$ we have the following fundamental result.

Theorem 5. Let $\mu: G \times M \rightarrow M$ be an effective and transitive left-action of Lie group $G$ on manifold $M$. Suppose $\omega=A(t) d t \in \Omega(\mathbb{R}, \mathfrak{g})$ is smooth at $t=0$. The unique solution of the initial value problem of Lie type

$$
\frac{d x}{d t}=\widehat{\boldsymbol{\mu}}(A(t))_{\left.\right|_{x(t)}}, \quad x(0)=q \in M
$$

is the function $x_{q}: \mathbb{R} \rightarrow M$ defined by

$$
x_{q}(t)=\mu(g(t), q),
$$

where $g: \mathbb{R} \rightarrow G$, is the fundamental solution. That is, $\delta(g)=\omega$ and $g(0)=e$.

Remark 3. This theorem reduces the construction of the solution of a system of Lie type to constructing the fundamental solution $g: \mathbb{R} \rightarrow G$ satisfying

$$
\frac{d g}{d t}=A(t) g(t), \quad g(0)=e .
$$

This problem can still be very challenging. Lie's approach is to simplify the problem by making use of any known solutions (Lie reduction). See [7] for a proof of Theorem 5.

To explain this, continuing with transitive and effective left-action $\mu: G \times M \rightarrow M$, suppose that $x_{1}(t), \ldots, x_{k}(t)$ are $k$ particular solutions of the Lie equation (10), satisfying

$$
x_{j}\left(t_{0}\right)=q_{j}, \quad 1 \leq j \leq k,
$$


for some collection of points $q_{j} \in M$. Denote by $G_{q}$ the isotropy subgroup of $q \in M$,

$$
G_{q}=\{g \in G \mid g \cdot q=q\}
$$

Let

$$
H=G_{q_{1}} \cap G_{q_{2}} \cap \cdots \cap G_{q_{k}},
$$

and let $\mathfrak{h}$ be the Lie algebra of $H$. Furthermore, let a curve $g_{0}: I \subseteq \mathbb{R} \rightarrow G$ be defined by

$$
\mu\left(g_{0}(t), q_{j}\right):=g_{0}(t) \cdot q_{j}=x_{j}(t) .
$$

Such a curve of group elements is defined up to a multiplication on the right by a curve in the the joint isotropy subgroup of the initial conditions. Constructing $g_{0}(t)$ involves the solution of algebraic equations with a potentially large solution space with no canonical choice of solution. In general one aims to find a solution $g_{0}(t)$ which passes through the identity at parameter value $t_{0}$ where initial conditions are to be posed.

Let $C^{\infty}(M, G)$ denote the set of smooth maps from $M$ to $G$. As in [7] define gauge transformations $\rho(h): \Omega(M, \mathfrak{g}) \rightarrow \Omega(M, \mathfrak{g})$ by

$$
\rho(h) \omega=\operatorname{Ad}(h) \omega+\delta(h), \quad \forall h \in C^{\infty}(M, G) .
$$

Theorem 6 (Lie reduction; see $[4,7]$ ). For any $\omega=A(t) d t \in \Omega(\mathbb{R}, \mathfrak{g})$,

1. $\omega_{1}=\rho\left(g_{0}(t)^{-1}\right) \omega \in \Omega(\mathbb{R}, \mathfrak{h})$.

2. If $g_{1}: I \subseteq \mathbb{R} \rightarrow H$ is an integral of $\omega_{1}$ then $g_{0}(t) g_{1}(t)$ is an integral of $\omega$.

\section{A.2.1 Application of Lie reduction to the Riccati equation}

The Cauchy problem for the integrable wave map system has been reduced to an initial value problem for Riccati equation (6) together with one quadrature. In this subsection we briefly illustrate the use of Lie reduction in the resolution of the general Riccati initial value problem. It is used in Section 3 of the paper. The standard action of the special linear group $G=\mathrm{SL}(2, \mathbb{R})$ on the real projective line induces a left-action on the real line

$$
\lambda_{g}(\xi):=\left[\begin{array}{ll}
a & b \\
c & d
\end{array}\right] \cdot \xi=\frac{a \xi+b}{c \xi+d}, \quad \xi \in \mathbb{R}
$$

the linear fractional or Möbius transformations.

With basis

$$
u_{-1}=\left[\begin{array}{ll}
0 & 1 \\
0 & 0
\end{array}\right], \quad u_{0}=\left[\begin{array}{cc}
1 & 0 \\
0 & -1
\end{array}\right], \quad u_{1}=\left[\begin{array}{ll}
0 & 0 \\
1 & 0
\end{array}\right]
$$

for $\mathfrak{s l}(2, \mathbb{R})$ it is easy to see that the anti-homomorphism $\widehat{\boldsymbol{\lambda}}: \mathfrak{s l}(2, \mathbb{R}) \rightarrow \mathfrak{X}(\mathbb{R})$ can be expressed

$$
\widehat{\boldsymbol{\lambda}}\left(\alpha_{0} u_{-1}+\alpha_{1} u_{0}+\alpha_{2} u_{1}\right)=\left(\alpha_{0}+2 \alpha_{1} x-\alpha_{2} x^{2}\right) \partial_{x} .
$$

Hence

$$
A(t)=\alpha_{0}(t) u_{-1}+\alpha_{1}(t) u_{0}+\alpha_{2}(t) u_{1}=\left[\begin{array}{cc}
\alpha_{1}(t) & \alpha_{0}(t) \\
\alpha_{2}(t) & -\alpha_{1}(t)
\end{array}\right]
$$

satisfies

$$
\widehat{\boldsymbol{\lambda}}(A(t))=\left(\alpha_{0}(t)+2 \alpha_{1}(t) x-\alpha_{2}(t) x^{2}\right) \partial_{x} .
$$


That is, $A(t) \subset \mathfrak{s l}(2, \mathbb{R})$ is associated with the Lie equation

$$
\frac{d x}{d t}=\alpha_{0}(t)+2 \alpha_{1}(t) x-\alpha_{2}(t) x^{2} .
$$

Now suppose that a solution $x_{0}(t)$ of equation (13) is known and suppose that $x_{0}(0)=0$. Solving equation (11) we obtain

$$
g_{0}(t)=\left[\begin{array}{cc}
1 & x_{0}(t) \\
0 & 1
\end{array}\right]
$$

The isotropy subgroup of $0 \in \mathbb{R}$ is

$$
H=G_{0}=\left[\begin{array}{cc}
a & 0 \\
c & a^{-1}
\end{array}\right]
$$

from which the initial value problem for the fundamental solution $g_{1}(t)$ of the reduced equation of Lie type can be deduced to be

$$
\frac{d g_{1}}{d t}=B(t) g_{1}(t), \quad g_{1}(0)=I_{2}
$$

where

$$
B(t) d t=\delta\left(g_{1}(t)\right)=\rho_{g_{0}(t)^{-1}}(\omega)=\left[\begin{array}{cc}
\alpha_{1}-x_{0} \alpha_{2} & 0 \\
-\alpha_{2} & -\left(\alpha_{1}-x_{0} \alpha_{2}\right)
\end{array}\right] d t .
$$

Since $g_{1}(t)$ is a curve in $H$, we have

$$
g_{1}(t)=\left[\begin{array}{cc}
\gamma_{1}(t) & 0 \\
\gamma_{2}(t) & \gamma_{1}(t)^{-1}
\end{array}\right]
$$

With $\beta(t)=\alpha_{1}-x_{0} \alpha_{2}$, (14) has solution, expressed in terms of quadrature

$$
\gamma_{1}(t)=\exp \int_{0}^{t} \beta(\tau) d \tau, \quad \gamma_{2}(t)=-\gamma_{1}(t)^{-1} \int_{0}^{t} \alpha_{2}(\tau) \gamma_{1}(\tau)^{2} d \tau .
$$

Finally, the unique solution of the initial value problem $x(0)=q \in \mathbb{R}$ for the Riccati equation can now be expressed in terms of quadrature:

$$
x(t)=g_{0}(t) g_{1}(t) \cdot q=\left[\begin{array}{cc}
\gamma_{1}(t)+x_{0}(t) \gamma_{2}(t) & x_{0}(t) / \gamma_{1}(t) \\
\gamma_{2}(t) & \gamma_{1}(t)^{-1}
\end{array}\right] \cdot q .
$$

That is,

$$
x(t)=\frac{\gamma_{1}(t) q}{\gamma_{2}(t) q+\gamma_{1}(t)^{-1}}+x_{0}(t) .
$$

Theorem 7. Let $x_{0}(t)$ be a known solution of a Riccati equation (13). Then by Lie reduction the construction of the fundamental solution is reducible to quadrature.

\section{Acknowledgements}

I'm grateful to the three anonymous referees for their close reading of the manuscript and for making suggestions which considerably improved the paper. I would like to acknowledge, with my thanks, the early involvement of Jordane Mathé for carefully working together through the calculations in Section 4 which formed a portion of his internship from the Ecole normale supérieure de Cachan, France. Much of the research for this paper was carried out while I was a Visiting Fellow at the Mathematical Sciences Institute of the Australian National University, Canberra. The hospitality of the MSI is gratefully acknowledged. In particular, I thank Mike Eastwood and the Differential Geometry Group for stimulating discussions. 


\section{References}

[1] Anderson I.M., Fels M.E., The Cauchy problem for Darboux integrable systems and non-linear d'Alembert formulas, SIGMA 9 (2013), 017, 22 pages, arXiv:1210.2370.

[2] Anderson I.M., Fels M.E., Vassiliou P.J., Superposition formulas for exterior differential systems, Adv. Math. 221 (2009), 1910-1963, arXiv:0708.0679.

[3] Baird P., Wood J.C., Harmonic morphisms between Riemannian manifolds, London Mathematical Society Monographs. New Series, Vol. 29, The Clarendon Press, Oxford University Press, Oxford, 2003.

[4] Bryant R.L., An introduction to Lie groups and symplectic geometry, in Geometry and Quantum Field Theory (Park City, UT, 1991), IAS/Park City Math. Ser., Vol. 1, Amer. Math. Soc., Providence, RI, 1995, 5-181, available at http://fds.duke.edu/db/aas/math/faculty/bryant/publications/9565.

[5] Cariñena J.F., de Lucas J., Lie systems: theory, generalisations, and applications, Dissertationes Math. 479 (2011), 162 pages, arXiv:1103.4166.

[6] Clelland J.N., Vassiliou P.J., A solvable string on a Lorentzian surface, arXiv:1303.0087.

[7] Dubrov B.M., Komrakov B.P., The constructive equivalence problem in differential geometry, Sb. Math. 191 (2000), 655-681.

[8] Eells J., Sampson J.H., Harmonic mappings of Riemannian manifolds, Amer. J. Math. 86 (1964), 109-160.

[9] Goursat E., Leçons sur l'intégration des équations aux dérivées partielles du seconde ordre á deux variables indépendent. II, Hermann, Paris, 1898.

[10] Gu C.H., On the Cauchy problem for harmonic maps defined on two-dimensional Minkowski space, Comm. Pure Appl. Math. 33 (1980), 727-737.

[11] Guest M.A., Harmonic maps, loop groups, and integrable systems, London Mathematical Society Student Texts, Vol. 38, Cambridge University Press, Cambridge, 1997.

[12] Hélein F., Wood J.C., Harmonic maps, in Handbook of Global Analysis, Editors D. Krupka, D. Saunders, Elsevier, Amsterdam, 2008, 417-491.

[13] Ivey T.A., Landsberg J.M., Cartan for beginners: differential geometry via moving frames and exterior differential systems, Graduate Studies in Mathematics, Vol. 61, Amer. Math. Soc., Providence, RI, 2003.

[14] Kostrigina O.S., Zhiber A.V., Darboux-integrable two-component nonlinear hyperbolic systems of equations, J. Math. Phys. 52 (2011), 033503, 32 pages.

[15] Ream R., Darboux integrability of wave maps into two-dimensional Riemannian manifolds, Masters degree thesis, Utah State University, 2008, available at http://digitalcommons.usu.edu/etd/203/.

[16] Shatah J., Struwe M., Geometric wave equations, Courant Lecture Notes in Mathematics, Vol. 2, New York University Courant Institute of Mathematical Sciences, New York, 1998.

[17] Tao T., Wave maps, http://www.math.ucla.edu/ tao/preprints/wavemaps.pdf.

[18] Vassiliou P.J., A constructive generalised Goursat normal form, Differential Geom. Appl. 24 (2006), 332-350, math.DG/0404377.

[19] Vassiliou P.J., Efficient construction of contact coordinates for partial prolongations, Found. Comput. Math. 6 (2006), 269-308, math.DG/0406234.

[20] Vassiliou P.J., Tangential characteristic symmetries and first order hyperbolic systems, Appl. Algebra Engrg. Comm. Comput. 11 (2001), 377-395.

[21] Vassiliou P.J., Vessiot structure for manifolds of $(p, q)$-hyperbolic type: Darboux integrability and symmetry, Trans. Amer. Math. Soc. 353 (2001), 1705-1739.

[22] Zakrzewski W.J., Low-dimensional sigma models, Adam Hilger Ltd., Bristol, 1989.

[23] Zhiber A.V., Sokolov V.V., Exactly integrable hyperbolic equations of Liouville type, Russ. Math. Surv. 56 (2001), no. 1, 61-101. 